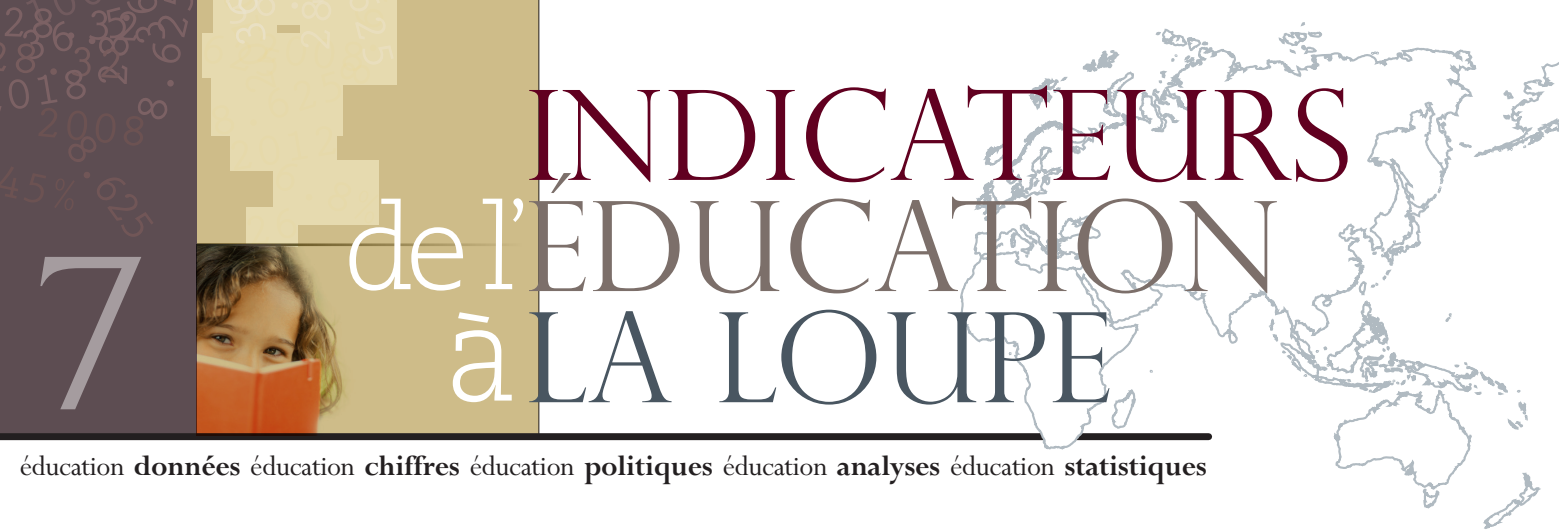

\title{
Dans quelle mesure les pays parviennent-ils à former les jeunes au niveau requis pour trouver un emploi et gagner leur vie?
}

- L'obtention d'un diplôme du deuxième cycle du secondaire (niveau CITE 3) est devenue la norme chez les jeunes des pays de l'OCDE. À I'heure actuelle, ce niveau de formation est considéré comme le minimum requis pour une intégration réussie sur le marché du travail et dans la société.

- Selon les données de 2010, dans les pays de l'OCDE, $19.1 \%$ des jeunes âgés de 25 à 34 ans dont le niveau de formation est inférieur au deuxième cycle du secondaire sont sans emploi, contre $9.8 \%$ des jeunes du même groupe d'âge diplômés du deuxième cycle du secondaire.

- Depuis 2004 jusque 2008, l'augmentation des taux d'obtention d'un diplôme du deuxième cycle du secondaire a coïncidé avec la diminution du nombre de jeunes âgés de 20 à 24 ans non scolarisés et sans emploi ; mais face à la crise économique, un diplôme du deuxième cycle du secondaire ne constitue plus une assurance suffisante contre le chômage et la pauvreté.

Dans de nombreux pays de l'OCDE, le deuxième cycle du secondaire devient le niveau minimum de formation...

L'évolution constante de la conjoncture économique et sociale alimente la demande de compétences plus élevées. Bien que de nombreux jeunes quittent le système d'éducation avant la fin du deuxième cycle du secondaire, il est clairement démontré que, dans les pays de l'OCDE, les élèves qui arrêtent leurs études sans diplôme du deuxième cycle du secondaire font en général face à d'importantes difficultés au moment d'entrer sur le marché du travail et, par la suite, pour y rester. Certains pays, tels que la Belgique (Communauté flamande) ou le Portugal, tentent d'accroître les possibilités d'obtenir ultérieurement un diplôme du deuxième cycle du secondaire grâce à des programmes de "seconde chance ». D'autres, comme les Pays-Bas, ont décidé de soumettre le versement des allocations familiales subventionnant la scolarité obligatoire à l'obtention d'un niveau minimum de formation.

Au cours des dernières décennies, la quasi-totalité des pays de l'OCDE ont connu une élévation spectaculaire du niveau de formation entre les générations. En moyenne, selon les données de 2010, $74 \%$ des individus âgés de 25 à 64 ans atteignent un niveau de formation égal ou supérieur au deuxième cycle du secondaire, proportion qui s'élève même à $82 \%$ chez les 25-34 ans. L'écart entre la génération des 25-34 ans et celle des 55-64 ans s'établit à 20 points de pourcentage, en moyenne, dans les pays de I'OCDE ; toutefois, au Chili, en Corée, en Espagne, en Grèce, en Irlande, en Italie et au Portugal, cet écart atteint, voire dépasse, 30 points de pourcentage.

Il existe différentes mesures pour rendre compte du niveau d'instruction d'une population ou d'un groupe d'âge donnés. Le niveau de formation correspond au pourcentage d'individus qui ont atteint un certain niveau d'enseignement, par exemple le deuxième cycle du secondaire (niveau CITE 3). Le taux d'obtention d'un diplôme correspond à l'estimation du pourcentage d'individus d'un groupe d'âge donné qui, au cours de leur vie, termineront avec succès une formation d'un certain niveau d'enseignement. Le taux de réussite correspond au pourcentage d'individus qui entament pour la première fois une formation d'un certain niveau d'enseignement et la terminent avec succès après un nombre d'années donné. 


\section{Pourcentage d'individus dont le niveau de formation est égal ou supérieur au deuxième cycle du secondaire ${ }^{1}$ (2010) En pourcentage, selon le groupe d'âge}

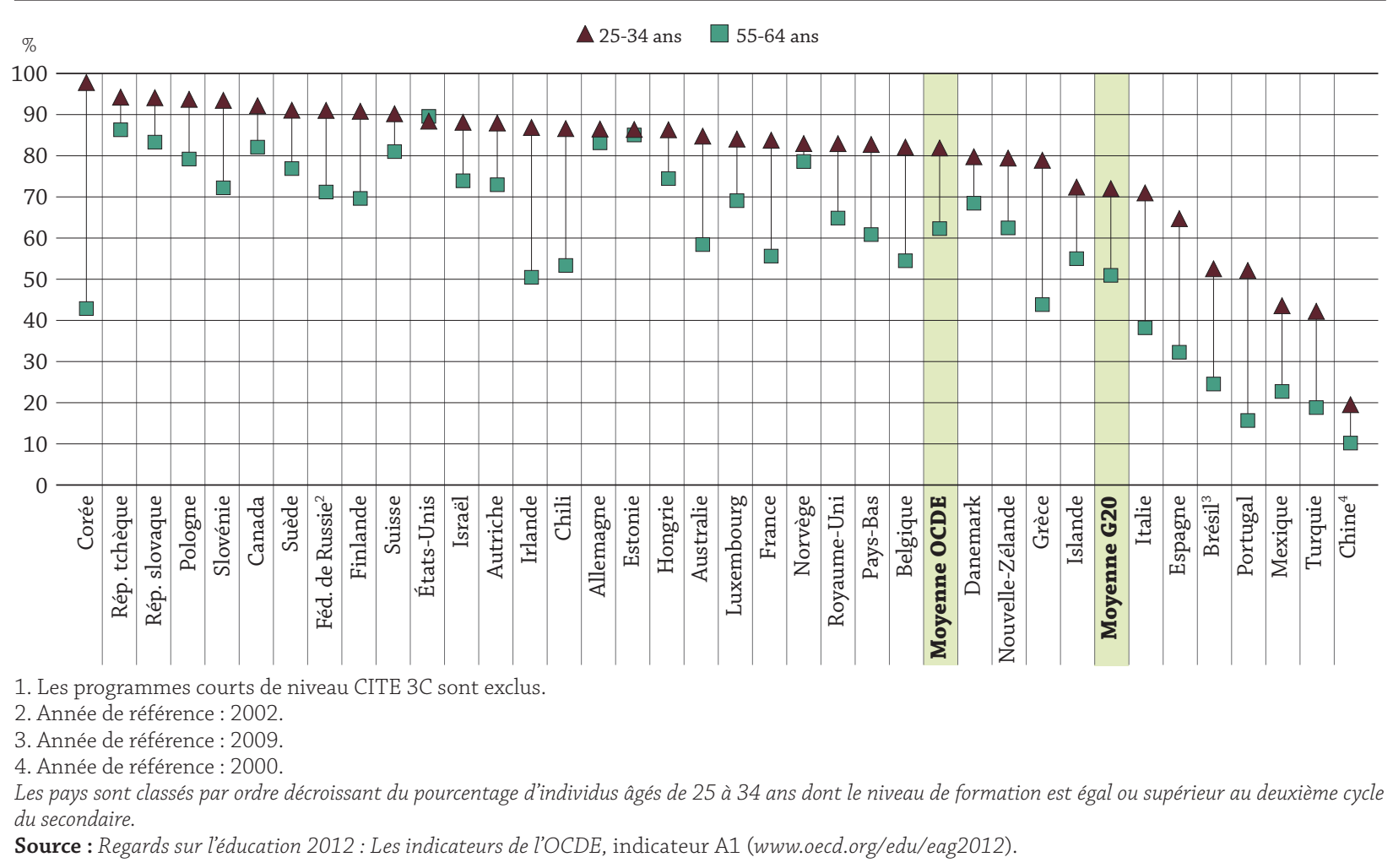

\section{... mais tous les pays ne parviennent pas à former leurs jeunes à ce niveau.}

La capacité d'un pays à permettre à ses jeunes d'obtenir un diplôme du deuxième cycle du secondaire constitue le facteur le plus déterminant de l'élévation du niveau de formation entre les générations. Dans la plupart des pays de l'OCDE, les taux d'obtention d'un premier diplôme du deuxième cycle du secondaire passent désormais la barre des $75 \%$, tandis que dans des pays tels que la Corée, la Finlande, la Grèce, I'IIlande, Israël, le Japon, le Portugal, le Royaume-Uni et la Slovénie, ils atteignent, voire dépassent, $90 \%$. Ces pays ont mis en œuvre des politiques efficaces afin d'augmenter le nombre de diplômés du deuxième cycle du secondaire. Par contraste, dans des pays tels que les États-Unis, le Luxembourg, le Mexique, la République tchèque, la Suède et la Turquie, ces taux restent inférieurs à $80 \%$.

Les individus dont le niveau de formation est inférieur au deuxième cycle du secondaire sont plus exposés au risque de chômage et de pauvreté...

Les indicateurs de l'éducation et de l'emploi montrent de façon systématique que plus le niveau de formation d'un individu est élevé, plus ce dernier est susceptible d'occuper un emploi. En moyenne, selon les données de 2010, le taux d'emploi des individus âgés de 25 à 64 ans diplômés du deuxième cycle du secondaire s'établit à 74 \%, contre $55 \%$ chez les individus qui n'ont pas atteint ce niveau d'enseignement. En outre, la probabilité d'occuper un emploi durable à plein temps augmente avec l'élévation du niveau de formation. En dépit des variations selon le groupe d'âge et le sexe, et de différences considérables entre les pays, ces données montrent de façon constante que l'obtention d'un diplôme du deuxième cycle du secondaire est devenue une condition préalable à l'amélioration des possibilités d'emploi. 
La faiblesse du niveau de formation accroît également le risque de pauvreté : 27 \% environ des individus dont le niveau de formation est inférieur au deuxième cycle du secondaire ont des revenus inférieurs à la moitié du niveau médian, soit une proportion supérieure d'environ 10 points de pourcentage à celle parmi les diplômés du deuxième cycle du secondaire. Cet écart de risque relatif de pauvreté est particulièrement marqué (plus de 15 points de pourcentage) en Autriche, au Luxembourg, en République slovaque et au Royaume-Uni, et culmine en Corée et aux États-Unis (20 points de pourcentage ou plus). En outre, cet écart est bien plus marqué chez les femmes que chez les hommes : dans les pays de l'OCDE, l'écart de pourcentage de femmes dont les revenus sont inférieurs à la moitié du niveau médian, selon qu'elles ont obtenu ou non un diplôme du deuxième cycle du secondaire, s'établit à 15 points de pourcentage, en moyenne, contre 7 points de pourcentages chez les hommes. Dans 8 pays, cet écart est supérieur à 20 points de pourcentage chez les femmes.

\section{... et les jeunes moins instruits constituent une population particulièrement à risque.}

Pour les jeunes hommes et femmes dont le niveau de formation est inférieur au deuxième cycle du secondaire, la transition entre les études et le marché du travail s'avère bien plus difficile ; par ailleurs, la capacité à intégrer ces individus moins instruits sur le marché du travail varie fortement entre les pays. Selon les données de 2010, parmi les individus non scolarisés et sans emploi, la proportion de jeunes âgés de 20 à 24 ans dont le niveau de formation est inférieur au deuxième cycle du secondaire est supérieure de 8 points de pourcentage à celle des jeunes du même groupe d'âge diplômés du deuxième cycle du secondaire. Toujours selon les données de 2010, en Espagne, en

Estonie, en France, en Irlande et en République slovaque, $25 \%$ au moins des jeunes âgés de 20

à 24 ans dont le niveau de formation est inférieur au deuxième cycle du secondaire sont non scolarisés et sans emploi. Par contraste, la Corée, le Danemark, le Mexique et les Pays-Bas sont parvenus à limiter le pourcentage d'individus peu instruits et sans emploi : dans ces pays, leur proportion varie entre $4.9 \%$ et $6.8 \%$.

\section{Évolution des taux d'obtention d'un diplôme et de la proportion d'individus} non scolarisés et sans emplois

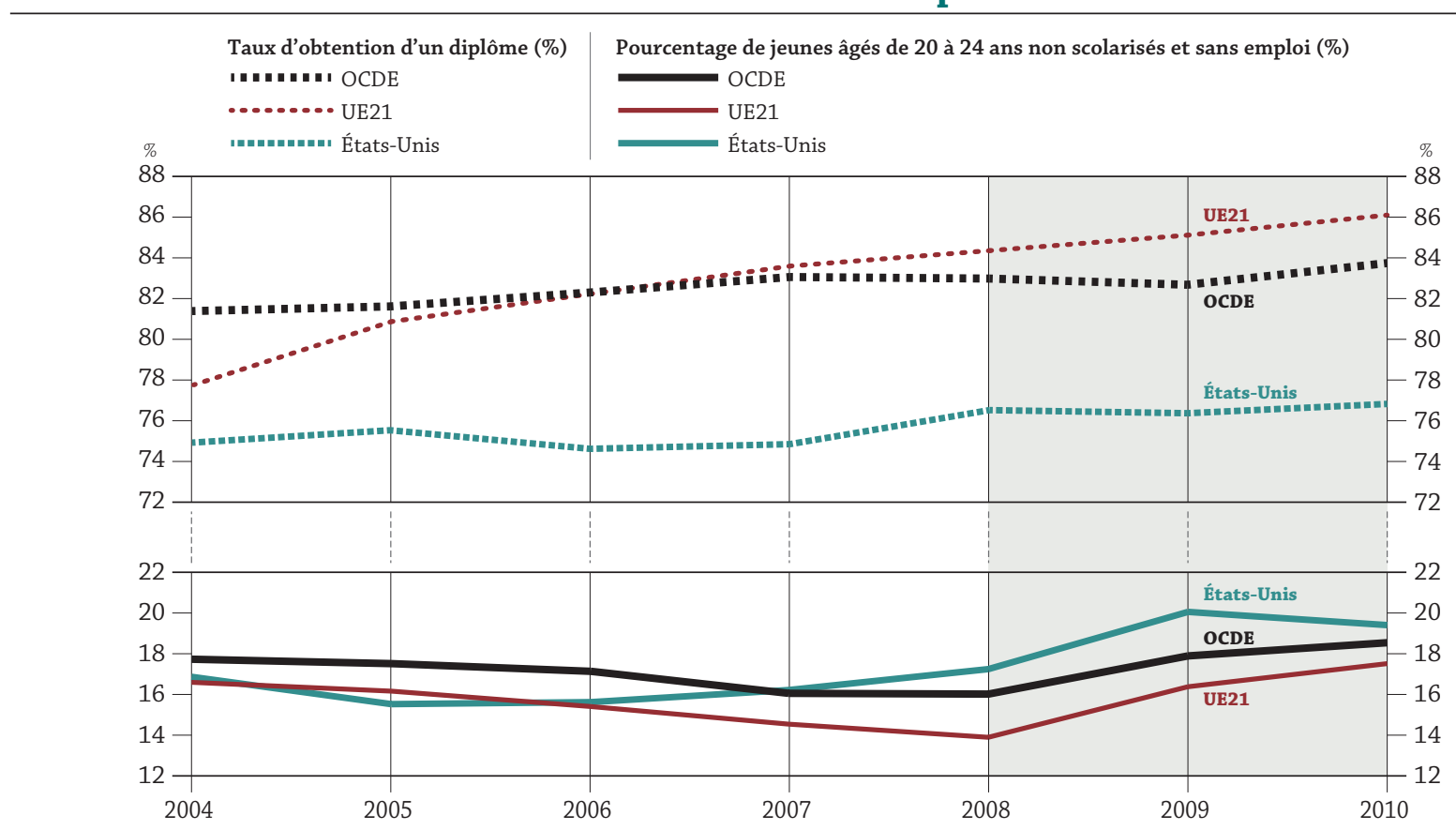

Source : Regards sur l'éducation 2012 : Les indicateurs de l'OCDE, indicateurs A2 et C5 (www.oecd.org/edu/eag2012). 
L'élévation du niveau de formation permet de minimiser ces risques...

L'évolution des taux d'obtention d'un diplôme du deuxième cycle du secondaire semble avoir eu un impact positif sur la taille de la population non scolarisée et sans emploi. Au cours des dix dernières années, les taux moyens d'obtention d'un diplôme ont connu une augmentation constante dans la plupart des pays de l'OCDE, notamment dans les pays de I'UE21'. La même tendance s'observe aux États-Unis, quoique dans une moindre mesure. Parallèlement, on a assisté au recul relatif de la proportion de jeunes non scolarisés et sans emploi chez les 20-24 ans, tout du moins jusqu'en 2008. Entre 2004 et 2008, les politiques menées dans les pays de l'UE21 se sont avérées particulièrement efficaces pour réduire le pourcentage de chômeurs dans ce groupe d'âge.

Aux États-Unis, la légère augmentation des taux d'obtention d'un diplôme entre 2004 et 2010 n'a pas été suffisante pour réduire de façon significative la taille de la population non scolarisée et sans emploi. La situation a également changé dans les pays de I'UE21 et de I'OCDE en 2008, avec l'accroissement soudain de cette population. La crise économique a clairement amoindri le rôle d'assurance contre le chômage que jouait le diplôme du deuxième cycle du secondaire. En effet, ce sont les individus bénéficiant d'un niveau de formation légèrement plus élevé qui occupent désormais les emplois qui revenaient traditionnellement aux individus moins instruits.

\section{... mais durant la récente crise économique, les bénéfices relatifs d'un diplôme du deuxième cycle du secondaire se sont amoindris.}

Les bénéfices associés à l'obtention d'un diplôme du deuxième cycle du secondaire sont-ils durables - même en cas de crise économique ? Les données tendancielles relatives à l'emploi et aux revenus suggèrent que, depuis le début de la crise en 2008, les bénéfices relatifs de l'obtention d'un diplôme du deuxième cycle du secondaire ont quelque peu diminué. En moyenne, dans les pays de l'OCDE, chez les 25-34 ans, l'écart de taux de chômage entre les jeunes dont le niveau de formation est inférieur au deuxième cycle du secondaire et les jeunes diplômés de l'enseignement tertiaire s'est accentué, passant de 9 à 13 points de pourcentage entre 2008 et 2010 ; dans les pays de l'UE21, cet écart s'est également creusé, passant de 11 à 16 points de pourcentage (durant cette période, il a atteint plus de 10 points de pourcentage en Estonie, en Irlande et en République slovaque).

Sous l'angle des revenus, la situation est tout autre. Entre 2008 et 2010, l'écart de revenus s'est creusé entre les diplômés de l'enseignement tertiaire et les individus dont le niveau de formation est inférieur au deuxième cycle du secondaire, augmentant de 9 points de pourcentage, en moyenne, dans les pays de l'OCDE ; toutefois, cette augmentation est presque entièrement imputable à l'élévation des revenus des individus les plus qualifiés. Selon les données disponibles, la crise a accéléré la polarisation de l'emploi en fonction des niveaux de compétences. Les individus dont le niveau de formation est inférieur au deuxième cycle du secondaire sont très vulnérables face au chômage, tandis que les diplômés du deuxième cycle du secondaire occupent des emplois moins bien rémunérés. Le diplôme de l'enseignement tertiaire s'impose donc désormais comme une condition préalable à l'obtention d'un emploi bien rémunéré.

1. La moyenne de I'UE21 correspond à la moyenne non pondérée des valeurs des 21 pays de l'OCDE membres de l'Union européenne dont les données sont disponibles, à savoir : I'Allemagne, l'Autriche, la Belgique, le Danemark, l'Espagne, l'Estonie, la Finlande, la France, la Grèce, la Hongrie, l'Irlande, I'Italie, le Luxembourg, les Pays-Bas, la Pologne, le Portugal, la République slovaque, la République tchèque, le Royaume-Uni, la Slovénie et la Suède.

Pour conclure Les individus dont le niveau de formation est inférieur au deuxième cycle du secondaire sont fortement exposés au risque de chômage et de pauvreté. Si la plupart des pays de l'OCDE sont parvenus à élargir les possibilités de formation afin de permettre à davantage de jeunes d'atteindre ce niveau minimum d'instruction, certains se sont heurtés à plus de difficultés. En 2008, le début de la crise économique a joué un rôle de révélateur : le diplôme du deuxième cycle du secondaire ne constitue plus, désormais, une assurance sans faille contre le chômage et l'emploi mal rémunéré.

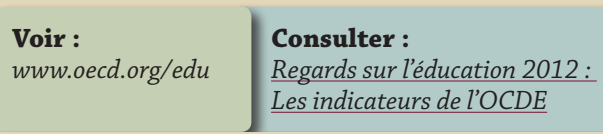

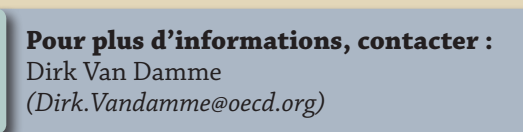

Prochain numéro :
Investissements public et privé
dans l'éducation Crédit photo: @ Ghislain \& Marie David de Lossy/Cultura/Getty Images 